\title{
Intermittent Fault Detection for Uncertain Networked Systems
}

\author{
Xiao He, ${ }^{1,2}$ Yanyan $\mathrm{Hu}^{3}$ and Kaixiang Peng ${ }^{4}$ \\ ${ }^{1}$ Department of Automation, TNList, Tsinghua University, Beijing 100084, China \\ ${ }^{2}$ School of Automation, Beijing Institute of Technology, Beijing 100081, China \\ ${ }^{3}$ Beijing Engineering Research Center of Industrial Spectrum Imaging, School of Automation and Electrical Engineering, \\ University of Science and Technology of Beijing, Beijing 100083, China \\ ${ }^{4}$ Key Laboratory for Advanced Control of Iron and Steel Process, School of Automation and Electrical Engineering, \\ University of Science and Technology of Beijing, Beijing 100083, China
}

Correspondence should be addressed to Kaixiang Peng; kaixiang@ustb.edu.cn

Received 12 September 2013; Accepted 25 September 2013

Academic Editor: Hongli Dong

Copyright (c) 2013 Xiao He et al. This is an open access article distributed under the Creative Commons Attribution License, which permits unrestricted use, distribution, and reproduction in any medium, provided the original work is properly cited.

\begin{abstract}
This paper investigates intermittent fault detection problem for a class of networked systems with multiple state delays and unknown input. Polytopic-type parameter uncertainty in the state-space model matrices is considered. A novel measurement model is employed to account for both the random measurement delays and the stochastic data missing (package dropout) phenomenon, which are typically resulted from the limited capacity of the communication networks. We aim to design an uncertainty-dependent fault detection filter such that, for all unknown input, all possible parameter uncertainties, and all incomplete measurements, the error between residual and weighted fault is made as small as possible. By converting the addressed robust fault detection problem into an alternative robust $H_{\infty}$ filtering problem of a certain Markovian jumping system (MJS), a sufficient condition for the existence of the desired robust fault detection filter is derived. A residual evaluation within an incremental form is brought forward to make the whole method suitable for intermittent fault detection. A numerical example is utilized to demonstrate the effectiveness of the proposed approach.
\end{abstract}

\section{Introduction}

For traditional control systems with point-to-point data transmission, a variety of methods have been proposed to deal with the modeling, identification, estimation, and control problems [1-3]. During the past decades, the rapid developments in network technologies have led to more and more feedback control systems with control loops closed via digital communication channels. Compared with the traditional point-to-point wiring, in networked systems, serial communication networks are used to exchange information (reference input, plant output, control input, etc.) among control system components (sensors, controller, actuators, etc.) [4]. The use of the communication channels can reduce the costs of cables and power, simplify the installation and maintenance of the whole system, and increase the reliability, so network-based analysis and designs have many industrial applications such as in automobiles, manufacturing plants, aircrafts, and HVAC systems. However, the insertion of the communication channels raises new interesting and challenging problems such as network-induced delays or packets dropout, see [4-6] for some representative works.

With the increasing demand for higher performance, higher safety, and reliability standards, fault detection and isolation (FDI) has been an active field of research over the past decades $[7,8]$. The main purpose of fault detection is to construct a residual signal which can then be compared with a predefined threshold. When the residual exceeds the threshold, the fault is detected and an alarm is generated. Among different approaches for residual generation, the modelbased approaches to FDI problems for dynamic systems have received more attention. For example, in [9], the $H_{\infty}$ norm of transfer function matrix from unknown input to residual has been designed to be small, while the $H_{\infty}$ norm (or the smallest nonzero singular value) of transfer function matrix from fault to residual has been guaranteed to be large. In [10], 
the error between residual and weighted fault has been made as small as possible, and then the FDI problem can be solved by using the $H_{\infty}$ filtering approach.

Due to the popularization of the using of network cables, it is necessary and interesting to consider the FDI problem for networked systems with network-induced delays or data missing, see $[11,12]$ and the references therein. Since networkinduced delays and data missing (dropout) phenomenon are inherently random and time-varying [13], they have been modeled in various probabilistic ways [14]. One of the attractive approaches is to use binary switching sequence viewed as a Bernoulli distributed white sequence taking on values of 0 and 1 , since such a representation is very effective to describe network-induced delays [15] or data missing [16]. Very recently, in [17], the network-induced delay and data dropout problems have been investigated in an integrated way within a unified framework and the robust filtering problem with polytopic uncertainties has been thoroughly studied. Note that in all the aforementioned results, it has been assumed that the delay or missing characteristics are statistically mutually independent from transfer to transfer. In [18], the fault detection problem for systems with missing measurements has been discussed by characterizing the residual dynamics by a discrete-time MJS. In [19], the diagnosis of intermittent faults in dynamic systems modeled as discrete event systems has been considered. So far, to the best of the authors' knowledge, the robust intermittent fault detection problems in the presence of parameter uncertainty for networked systems with simultaneous measurement delays and data missing have not been fully investigated, which constitutes the main focus of this paper.

In this paper, intermittent fault detection problem for a class of uncertain networked systems with multiple state delays and incomplete measurement is investigated. A sequence varying in a Markov fashion is employed in the measurement model so that both the measurement delays and data missing can be simultaneously represented. Polytopictype parameter uncertainty in state-space model matrices is considered. After augmenting the state, the addressed robust fault detection problem is converted to an equivalent robust $H_{\infty}$ filtering problem for a certain Markovian jumping system (MJS), and a sufficient condition for the existence of the desired robust fault detection filter is brought forward. By introducing the new residual evaluation function within an incremental form, the proposed method can detect the possible intermittent fault.

Notation. The notations used throughout the paper are fairly standard. $\mathbb{R}^{n}$ and $\mathbb{R}^{n \times m}$ denote, respectively, the $n$-dimensional Euclidean space and the set of all $n \times m$ real matrices. $P>0$ means that $P$ is real symmetric and positive definite. The subscript " $T$ " denotes the matrix transpose. $\operatorname{Pr}\{\cdot\}$ represents the occurrence probability of the event ".", and when $x$ and $y$ are both stochastic variables, $\mathbb{E}\{x\}$ stands for the mathematical expectation of $x . l_{2}[0, \infty)$ is the space of all square-summable vector functions over $[0, \infty)$, with $\|x\|$ being the standard $l_{2}$ norm of $x$, that is, $\|x\|=\left(x^{T} x\right)^{1 / 2}$. $\mathbf{R H}_{\infty}$ is the set of proper and stable rational functions with real coefficients. In symmetric block matrices, we use
"*" to represent a term that is induced by symmetry, and $\operatorname{diag}\{\ldots\}$ stands for a block-diagonal matrix. Matrices, if their dimensions are not explicitly stated, are assumed to be compatible for algebraic operations and, sometimes, the arguments of a function will be omitted in the analysis when no confusion can arise.

\section{Problem Formulation and Preliminaries}

Consider the following class of discrete-time linear networked systems with multiple delays in the state:

$$
\begin{gathered}
x_{k+1}=A_{0} x_{k}+\sum_{i=1}^{q} A_{i} x_{k-i}+B_{w} w_{k}+B_{f} f_{k}, \\
y_{k}=\delta\left(\tau_{k}, 0\right) C_{0} x_{k}+\sum_{i=1}^{q} \delta\left(\tau_{k}, i\right) C_{i} x_{k-i}+D w_{k}, \\
x_{k}=\varphi_{k}, \quad k=-q,-q+1, \ldots, 0,
\end{gathered}
$$

where $x_{k} \in \mathbb{R}^{n}$ stands for the state vector, $w_{k} \in \mathbb{R}^{p}$ is the unknown input belonging to $l_{2}[0, \infty)$, and $f_{k} \in \mathbb{R}^{l}$ is the fault to be detected. $1 \leq i \leq q(q \geq 1)$ are integer time delays. $y_{k} \in \mathbb{R}^{m}$ is the measured output vector, which may contain random communication delays and stochastic data missing induced by the limited capacity of the communication networks. All system matrices in (1) are assumed to have appropriate dimensions. $\varphi_{k}$ is a given real initial sequence on $[-q, 0]$, and $\delta(\cdot, \cdot)$ stands for the Kronecker delta; that is,

$$
\delta(j, l)= \begin{cases}0, & \text { if } j \neq l, \\ 1, & \text { if } j=l .\end{cases}
$$

Furthermore, $\tau_{k}$ is a stochastic variable whose role is to determine, at time $k$, the size of the occurred delay as well as the possibility of data missing. In this paper, $\left\{\tau_{k}\right\}$ is assumed to be a discrete-time homogeneous Markov chain taking values in the following finite state space:

$$
\Xi=\{-1,0, \ldots, q\}
$$

and stationary transition probability matrix $\Lambda=\left[\lambda_{i j}\right]$, where

$$
\lambda_{i j}=\operatorname{Pr}\left\{\tau_{k+1}=j \mid \tau_{k}=i\right\} .
$$

Remark 1 . The assumption that the switching between difference modes abides by a Markovian chain seems realistic since, in network-based signal transmissions, time delays and data dropouts typically occur in a batch mode, and the status of the network varies slower than the sampling period and the characteristics of the network at a certain time is usually dependent on the superior time instant. The transition from one mode to another may obey certain probability distribution, and our "Markov jumping" assumption of $\left\{\tau_{k}\right\}$ describes this phenomenon properly.

Remark 2. In our measurement model, the event $\left\{\tau_{k}=0\right\}$ means that the measurements are ideally transmitted over the network without any delays or data missing, the event $\left\{\tau_{k}=i\right\}$ 
( $1 \leq i \leq q)$ corresponds to the case that the $i$-step measurement delay occurs. Without loss of generality and for the convenience in denoting the transition probability matrix, we set the state in the state space of the Markov chain corresponding to the measurement missing situation to -1 , so $\left\{\tau_{k}=-1\right\}$ means that the measurements are missing and $y_{k}$ consists of pure noise only.

In this paper, we are interested in the problem of robust fault detection for uncertain system described by (1) with incomplete measurements. The system matrices $A_{0}, A_{1}, \ldots$, $A_{q}, B_{w}, B_{f}, C_{0}, C_{1}, \ldots, C_{q}, D$ are assumed to be uncertain but belong to a known convex compact set of polytopic type,that is,

$$
\Omega:=\left(A_{0}, A_{1}, \ldots, A_{q}, B_{w}, B_{f}, C_{0}, C_{1}, \ldots, C_{q}, D\right) \in \Theta,
$$

where $\Theta$ is a given convex bounded polyhedral domain described by $v$ vertices as follows:

$$
\Theta:=\left\{\Omega \mid \Omega=\sum_{r=1}^{v} \alpha_{r} \Omega_{r} ; \sum_{r=1}^{v} \alpha_{r}=1, \alpha_{r} \geq 0\right\},
$$

where $\Omega_{r}:=\left(A_{0 r}, A_{1 r}, \ldots, A_{q r}, B_{w r}, B_{f r}, C_{0 r}, C_{1 r}, \ldots, C_{q r}\right.$, $\left.D_{r}\right), r=1, \ldots, v$, denotes the $r$ th vertex of the polytope. form:

Consider a full-order fault detection filter of the following

$$
\begin{gathered}
\tilde{x}_{k+1}=G\left(\tau_{k}\right) \tilde{x}_{k}+K\left(\tau_{k}\right) y_{k}, \\
r_{k}=L\left(\tau_{k}\right) \tilde{x}_{k},
\end{gathered}
$$

where $\tilde{x}_{k} \in \mathbb{R}^{n}$ is the filter state vector and $r_{k} \in \mathbb{R}^{l}$ is the so-called residual that is compatible with the fault vector $f_{k}$. For $\tau_{k}=i \in \Xi$, we denote matrices $G\left(\tau_{k}\right), K\left(\tau_{k}\right)$, and $L\left(\tau_{k}\right)$ as $G_{i}=G\left(\tau_{k}=i\right), K_{i}=K\left(\tau_{k}=i\right)$, and $L_{i}=L\left(\tau_{k}=i\right)$. Our main aim is to make the error between residual $r_{k}$ and fault signal $f_{k}$ as small as possible.

For the purpose of fault detection, it is not necessary to estimate the fault $f_{k}$. Sometimes one is more interested in the fault signal of a certain frequency interval, which can be formulated as the weighted fault as follows:

$$
\widehat{f}(z)=T_{f}(z) f(z),
$$

where $T_{f}(z) \in \mathbf{R H}_{\infty}$ is a prescribed weighting matrix.

Remark 3. Similar to [10], the introduction of a suitable weighting matrix $T_{f}(z)$ can limit the frequency interval of interest, and the system performance can then be improved. In fact, the use of weighted fault $\widehat{f}(z)$ is more general than using the original fault $f(z)$, because if we impose $T_{f}(z)=I$, we can obtain $\widehat{f}(z)=f(z)$.

Suppose a minimal realization of $\widehat{f}(z)=T_{f}(z) f(z)$ is

$$
\begin{aligned}
& \widehat{x}_{k+1}=A_{t} \widehat{x}_{k}+B_{t} f_{k}, \\
& \widehat{f}_{k}=C_{t} \widehat{x}_{k}+D_{t} f_{k},
\end{aligned}
$$

where $\widehat{x}_{k} \in \mathbb{R}^{\hat{n}}$ is the weighted fault state, $f_{k} \in \mathbb{R}^{l}$ is the original fault, and $\widehat{f}_{k} \in \mathbb{R}^{l}$ is the weighted fault. $A_{t}, B_{t}, C_{t}$, and $D_{t}$ are assumed to be known real constant matrices with appropriate dimensions.

By defining

$$
\begin{gathered}
\zeta_{k}=\left[\begin{array}{lll}
w_{k}^{T} & f_{k}^{T}
\end{array}\right]^{T}, \quad \tilde{r}_{k}=r_{k}-\widehat{f}_{k}, \\
\bar{x}_{k}=\left[\begin{array}{lll}
x_{k-1}^{T} & \cdots & x_{k-q}^{T}
\end{array}\right]^{T}, \quad \eta_{k}=\left[\begin{array}{llll}
x_{k}^{T} & \bar{x}_{k}^{T} & \tilde{x}_{k}^{T} & \widehat{x}_{k}^{T}
\end{array}\right]^{T}
\end{gathered}
$$

and again, denoting matrices $\widetilde{A}\left(\tau_{k}\right), \widetilde{B}\left(\tau_{k}\right), \widetilde{C}\left(\tau_{k}\right)$ and $\widetilde{D}\left(\tau_{k}\right)$ as $\widetilde{A}_{i}=\widetilde{A}\left(\tau_{k}=i\right), \widetilde{B}_{i}=\widetilde{B}\left(\tau_{k}=i\right), \widetilde{C}_{i}=\widetilde{C}\left(\tau_{k}=i\right)$, and $\widetilde{D}_{i}=\widetilde{D}\left(\tau_{k}=i\right)$, we have the overall fault detection dynamics governed by the following system:

$$
\begin{aligned}
& \eta_{k+1}=\widetilde{A}_{i} \eta_{k}+\widetilde{B}_{i} \zeta_{k}, \\
& \widetilde{r}_{k}=\widetilde{C}_{i} \eta_{k}+\widetilde{D}_{i} \zeta_{k},
\end{aligned}
$$

where

$$
\begin{aligned}
& \widetilde{A}_{i}=\left[\begin{array}{cc}
\widehat{A}_{i} & 0 \\
0 & A_{t}
\end{array}\right], \quad \widetilde{B}_{i}=\left[\begin{array}{c}
\widehat{B}_{i} \\
\widehat{B}_{t}
\end{array}\right], \\
& \widetilde{C}_{i}=\left[\begin{array}{ll}
\widehat{C}_{i} & -C_{t}
\end{array}\right], \quad \widetilde{D}_{i}=\left[\begin{array}{ll}
0 & -D_{t}
\end{array}\right], \\
& \widehat{A}_{i}=\left[\begin{array}{ccc}
A_{0} & A_{d} & 0 \\
\widehat{A}_{21} & \widehat{A}_{22} & 0 \\
\delta(i, 0) K_{0} C_{0} & K_{i} C_{i} e_{i} & G_{i}
\end{array}\right], \\
& \widehat{B}_{i}=\left[\begin{array}{cc}
B_{w} & B_{f} \\
0_{q n \times p} & 0_{q n \times l} \\
K_{i} D & 0
\end{array}\right], \quad \widehat{B}_{t}=\left[\begin{array}{ll}
0 & B_{t}
\end{array}\right] \text {, } \\
& \widehat{A}_{21}=\left[\begin{array}{c}
I_{n} \\
0_{(q-1) n \times n}
\end{array}\right], \quad \widehat{A}_{22}=\left[\begin{array}{cc}
0 & 0 \\
I_{(q-1) n \times(q-1) n} & 0
\end{array}\right] \text {, } \\
& A_{d}=\left[\begin{array}{lll}
A_{1} & \cdots & A_{q}
\end{array}\right], \\
& e_{i}=\left[\begin{array}{llll}
\delta(i, 1) I_{n \times n} & \cdots & \delta(i, q) I_{n \times n}
\end{array}\right], \\
& \widehat{C}_{i}=\left[\begin{array}{lll}
0_{l \times n} & 0_{l \times q n} & L_{i}
\end{array}\right], \quad \widehat{D}_{i}:=\widetilde{D}_{i} .
\end{aligned}
$$

After the above manipulations, the admissible sensor delays and data missing can be reformulated as the jumping parameters of a Markovian jumping system (11) with the same transition probability matrix $\Lambda$.

The matrices $\widetilde{A}_{i}, \widetilde{B}_{i}, \widetilde{C}_{i}$, and $\widetilde{D}_{i}, i \in \Xi$, are uncertain, but they belong to prescribed matrix polytopes $\widetilde{\Omega}_{i}:=$ $\left(\widetilde{A}_{i}, \widetilde{B}_{i}, \widetilde{C}_{i}, \widetilde{D}_{i},\right) \in \widetilde{\Theta}_{i}$, where $\widetilde{\Theta}_{i}$ are given convex bounded polyhedral domain described by $v$ vertices as follows:

$$
\widetilde{\Theta}_{i}:=\left\{\widetilde{\Omega}_{i} \mid \widetilde{\Omega}_{i}=\sum_{r=1}^{v} \alpha_{r} \widetilde{\Omega}_{i r} ; \sum_{r=1}^{v} \alpha_{r}=1, \alpha_{r} \geq 0\right\},
$$


and $\widetilde{\Omega}_{i r}=\left(\widetilde{A}_{i r}, \widetilde{B}_{i r}, \widetilde{C}_{i r}, \widetilde{D}_{i r}\right)$ denotes the $r$ th vertices of the polytopes, where

$$
\begin{aligned}
& \widetilde{A}_{i r}=\left[\begin{array}{cc}
\widehat{A}_{i r} & 0 \\
0 & A_{t}
\end{array}\right], \quad \widetilde{B}_{i r}=\left[\begin{array}{c}
\widehat{B}_{i r} \\
\widehat{B}_{t}
\end{array}\right], \\
& \widetilde{C}_{i r}=\left[\begin{array}{ll}
\widehat{C}_{i} & -C_{t}
\end{array}\right]=\widetilde{C}_{i}, \\
& \widetilde{D}_{i r}=\left[\begin{array}{ll}
0 & -D_{t}
\end{array}\right]=\widehat{D}_{i r}, \\
& \widehat{A}_{i r}=\left[\begin{array}{ccc}
A_{0 r} & A_{d r} & 0 \\
\widehat{A}_{21} & \widehat{A}_{22} & 0 \\
\delta(i, 0) K_{0} C_{0 r} & K_{i} C_{i r} e_{i} & G_{i}
\end{array}\right] \text {, } \\
& \widehat{B}_{i r}=\left[\begin{array}{cc}
B_{w r} & B_{f r} \\
0_{q n \times p} & 0_{q n \times l} \\
K_{i} D_{r} & 0
\end{array}\right], \\
& A_{d r}=\left[\begin{array}{lll}
A_{1 r} & \cdots & A_{q r}
\end{array}\right] \text {. }
\end{aligned}
$$

Matrices $\widehat{A}_{21}, \widehat{A}_{22}, e_{i}, \widehat{B}_{t}, \widetilde{C}_{i}, \widehat{C}_{i}, \widetilde{D}_{i}$, and $\widehat{D}_{i}$ are the same as defined in (12). Note that from (14), matrices $\widetilde{A}_{i r}$, $\widetilde{B}_{i r}, \widetilde{C}_{i r}$, and $\widetilde{D}_{i r}$ are affinely dependent on the matrices $A_{0 r}, A_{1 r}, \ldots, A_{q r}, B_{w r}, B_{f r}, C_{0 r}, C_{1 r}, \ldots, C_{q r}, D_{r}$, then for any $i \in \Xi$, the uncertainty polytopes (13) have the same number of vertices $v$, as well as the same combination coefficients $\alpha_{r}$ with (6), but different vertices for different Markovian model $i$. MJSs.

Recall the following definition of mean square stability for

Definition 4 (see [20]). System (11) with $\zeta_{k}=0$ is said to be mean square stable if

$$
\mathbb{E}\left\{\left\|\eta_{k}\right\|^{2}\right\} \longrightarrow 0, \quad \text { as } k \longrightarrow \infty
$$

for any initial condition $\eta_{0}$ and initial distribution $\tau_{0} \in \Xi$.

We further introduce the following definition.

Definition 5. System (11) with uncertain $\widetilde{\Omega}_{i} \in \widetilde{\Theta}_{i}, i \in \Xi$ (resp., $\Lambda \in \Pi)$ is robust mean square stable if (11) is mean square stable for every $\widetilde{\Omega}_{i} \in \widetilde{\Theta}_{i}, i \in \Xi$ (resp., $\Lambda \in \Pi$ ).

Assumption 6. System (1) with $w_{k}=0$ and $f_{k}=0$ is assumed to be robust mean square stable.

With Definition 5, we can transform the robust fault detection filter design problem of system (1) to a robust $H_{\infty}$ filtering problem for MJS (11). What we need to do here is to find the filter parameters $G_{i}, K_{i}$ and $L_{i}(i \in \Xi)$ such that the augmented fault detection dynamics (11) is robust mean square stable and the infimum of $\gamma$ is made small in the feasibility of the following:

$$
\sup _{\zeta_{k} \neq 0} \frac{\mathbb{E}\left\{\left\|\widetilde{r}_{k}\right\|^{2}\right\}}{\left\|\zeta_{k}\right\|^{2}}<\gamma^{2}, \quad \gamma>0 .
$$

We further adopt a residual evaluation stage including an incremental evaluation function $J(k)$ and a threshold $J_{\text {th }}$ of the following form:

$$
\begin{aligned}
& J^{L}(k)=\left\{\sum_{h=k-L}^{k} r_{h}^{T} r_{h}\right\}^{1 / 2}, \\
& J_{\text {th }}^{L}=\sup _{w_{k} \in l_{2}, f_{k}=0} \mathbb{E}\left\{J^{L}(k)\right\},
\end{aligned}
$$

where $r_{1-L}=r_{2-L}=\cdots=r_{-1}=r_{0}=0$ and $L$ denotes the length of time window for evaluation function.

Based on (17), the occurrence of faults can be detected by comparing $J(k)$ with $J_{\text {th }}$ according to the following rule:

$$
\begin{aligned}
& J^{L}(k)>J_{\text {th }}^{L} \Longrightarrow \text { with faults } \Longrightarrow \text { alarm, } \\
& J^{L}(k) \leq J_{\text {th }}^{L} \Longrightarrow \text { no faults } \Longrightarrow \text { do nothing. }
\end{aligned}
$$

Remark 7. By introducing the residual evaluation function with an incremental form (17), one can detect the possible intermittent fault for an uncertain networked system by analyzing the residual signal once it is generated by the fault detection filter (7). The reason is that the residual evaluation signal (11) is decreased to a small value over time once the fault disappears. This nature makes the proposed method in this paper be used for intermittent fault detection.

\section{Fault Detection Filter Design}

In this section, we shall discuss the robust fault detection filter design problem of system (1), under the existence of parameter uncertainty (13). We introduce the following lemma which is useful in deriving our main results in the sequel.

Lemma 8. Consider system (1) with system uncertainty (5), for a given fault detection filter of the form (7), the augmented dynamic (11) is robust mean square stable and satisfies the constraint (16), if there exist matrices $P_{i r} \in \mathbb{R}^{(q+2) n}, i \in \Xi, r=$ $1, \ldots, v, P_{t} \in \mathbb{R}^{\widehat{n}}$, and $Q_{i} \in \mathbb{R}^{(q+2) n}$ such that the following LMIs

$$
\left[\begin{array}{cccccc}
-P_{i r} & \widehat{A}_{i r}^{T} Q_{i}^{T} & 0 & \widehat{C}_{i}^{T} & 0 & 0 \\
* & -Q_{i}-Q_{i}^{T}+\mathscr{P}_{r}^{T} \mathcal{S}_{i} & Q_{i} \widehat{B}_{i r} & 0 & 0 & 0 \\
* & * & -\gamma^{2} I & \widehat{D}_{i}^{T} & 0 & \widehat{B}_{t}^{T} P_{t} \\
* & * & * & -I & -C_{t} & 0 \\
* & * & * & * & -P_{t} & A_{t}^{T} P_{t} \\
* & * & * & * & * & -P_{t}
\end{array}\right]<0
$$

hold for all $i \in \Xi, r=1, \ldots, v$, where $\widehat{A}_{i r}, \widehat{B}_{i r}, \widehat{C}_{i}$, and $\widehat{D}_{i}$ are defined in (14), $\widehat{B}_{t}$ is defined in (12), $A_{t}, B_{t}, C_{t}, D_{t}$ are defined in (9) and,

$$
\begin{gathered}
\mathscr{P}_{r}=\left[\begin{array}{lll}
P_{(-1) r} & \cdots & P_{q r}
\end{array}\right]^{T}, \\
\mathcal{S}_{i}=\left[\begin{array}{lll}
\lambda_{i(-1)} I_{(q+2) n} & \cdots & \lambda_{i q} I_{(q+2) n}
\end{array}\right]^{T} .
\end{gathered}
$$


Proof. Considering the structure of $\widetilde{A}_{i}, \widetilde{B}_{i}, \widetilde{C}_{i}$, and $\widetilde{D}_{i}$, and imposing

$$
\widetilde{P}_{i}=\left[\begin{array}{cc}
P_{i} & 0 \\
0 & P_{t}
\end{array}\right]
$$

for all $i \in \Xi$, it can be concluded from the bounded real Lemma in [21] that a sufficient condition is that there exist matrices $P_{i} \in \mathbb{R}^{(q+2) n}, i \in \Xi, P_{t} \in \mathbb{R}^{\widehat{n}}$ such that the following LMIs

$$
\left[\begin{array}{cccccc}
-P_{i} & \widehat{A}_{i}^{T} \mathscr{P}^{T} \mathcal{S}_{i} & 0 & \widehat{C}_{i}^{T} & 0 & 0 \\
* & -\mathscr{P}^{T} \mathcal{S}_{i} & \mathscr{P}^{T} \mathcal{S}_{i} \widehat{B}_{i} & 0 & 0 & 0 \\
* & * & -\gamma^{2} I & \widehat{D}_{i}^{T} & 0 & \widehat{B}_{t}^{T} P_{t} \\
* & * & * & -I & -C_{t} & 0 \\
* & * & * & * & -P_{t} & A_{t}^{T} P_{t} \\
* & * & * & * & * & -P_{t}
\end{array}\right]<0
$$

hold for any $i \in \Xi$, where $\widehat{A}_{i}, \widehat{B}_{i}, \widehat{C}_{i}, \widehat{D}_{i}$, and $\widehat{B}_{t}$ are defined in (12), $A_{t}, B_{t}, C_{t}$, and $D_{t}$ are defined in (9), $\mathcal{S}_{i}$ is the same defined in (21) and

$$
\mathscr{P}=\left[\begin{array}{lll}
P_{(-1)} & \cdots & P_{q}
\end{array}\right]^{T} .
$$

Following the steps as the proof of Theorem 1 in [22], it can be shown that LMIs (23) are feasible if and only if there exist matrices $P_{i} \in \mathbb{R}^{(q+2) n}, Q_{i} \in \mathbb{R}^{(q+2) n}, i \in \Xi$, and $P_{t} \in$ $\mathbb{R}^{\widehat{n}}$ satisfying

$$
\left[\begin{array}{cccccc}
-P_{i} & \widehat{A}_{i}^{T} Q_{i}^{T} & 0 & \widehat{C}_{i}^{T} & 0 & 0 \\
* & -Q_{i}-Q_{i}^{T}+\mathscr{P}^{T} \mathcal{S}_{i} & Q_{i} \widehat{B}_{i} & 0 & 0 & 0 \\
* & * & -\gamma^{2} I & \widehat{D}_{i}^{T} & 0 & \widehat{B}_{t}^{T} P_{t} \\
* & * & * & -I & -C_{t} & 0 \\
* & * & * & * & -P_{t} & A_{t}^{T} P_{t} \\
* & * & * & * & * & -P_{t}
\end{array}\right]<0 .
$$

We are now in the position to prove that for system (1) with uncertainty (13), (20) ensures the robust mean square stable as well as the constraint (16) of the augmented dynamic (11). For an arbitrary fixed uncertain system with system matrices $\Omega$, one can always find a set of coefficients $\alpha_{r} \geq$ $0, r=1, \ldots, v$, such that both (6) and (13) hold. Note that LMIs $(20)$ are affine in the matrices $P_{i r}, \widehat{A}_{i r}, \widehat{B}_{i r}, \widehat{C}_{i}$, and $\widehat{D}_{i}$, multiplying suitable inequalities of (20) by appropriate scalars $\alpha_{r}$ and summing up, it can be readily shown that (25) holds for every $\widetilde{\Omega}_{i}$ with a matrix $P_{i}(\alpha)=\sum_{r=1}^{v} \alpha_{r} P_{i r}, i=$ $-1, \ldots, q$. By using the Bounded Real Lemma in [21], it follows that system (1) is robust mean square stable and (16) is satisfied. This concludes the proof.

Next, we give the robust fault detection filter design result for system (1) with system uncertainty (13).

Theorem 9. Consider system (1) with uncertain matrices (13), let $\gamma>0$ be a given scalar, there exists an admissible full-order robust fault detection filter of the form (7) ensuring that the overall augmented dynamics (11) is robust mean square stable and the constraint (16) is satisfied, if there exist matrices $0<$ $X_{i r}^{T}=X_{i r} \in \mathbb{R}^{(q+2) n \times(q+2) n}, S_{i} \in \mathbb{R}^{n \times n}, Z_{i} \in \mathbb{R}^{n \times n}, Y_{i} \in$ $\mathbb{R}^{n \times n}, \bar{G}_{i} \in \mathbb{R}^{n \times n}, \bar{K}_{i} \in \mathbb{R}^{n \times m}, \bar{L}_{i} \in \mathbb{R}^{l \times n}, M_{i} \in \mathbb{R}^{q n \times q n}, i=$ $-1, \ldots, q, r=1, \ldots, v$, and $0<P_{t}^{T}=P_{t} \in \mathbb{R}^{\hat{n} \times \hat{n}}$, such that the following LMIs

$$
\left[\begin{array}{cccccc}
-X_{i r} & \Phi_{12} & 0 & \Phi_{14} & 0 & 0 \\
* & \Phi_{22} & \Phi_{23} & 0 & 0 & 0 \\
* & * & -\gamma^{2} I & \widehat{D}_{i r}^{T} & 0 & \widehat{B}_{t}^{T} P_{t} \\
* & * & * & -I & -C_{t} & 0 \\
* & * & * & * & -P_{t} & A_{t}^{T} P_{t} \\
* & * & * & * & * & -P_{t}
\end{array}\right]<0
$$

$$
\begin{gathered}
\Phi_{12}=\left[\begin{array}{ccc}
A_{0 r}^{T} Z_{i}^{T} & {\left[\begin{array}{ll}
I_{n \times n} & 0_{n \times(q-1) n}
\end{array}\right] M_{i}^{T}} & A_{0 r}^{T} Y_{i}^{T}+\delta(i, 0) C_{0 r}^{T} \bar{K}_{0}^{T}+\bar{G}_{i}^{T} \\
A_{d r}^{T} Z_{i}^{T} & {\left[\begin{array}{cc}
0_{(q-1) n \times n} & I_{(q-1) n \times(q-1) n} \\
0_{n \times n} & 0_{n \times(q-1) n}
\end{array}\right] M_{i}^{T}} & A_{d r}^{T} Y_{i}^{T}+e_{i}^{T} C_{i r}^{T} \bar{K}_{i}^{T} \\
A_{0 r}^{T} Z_{i}^{T} & {\left[\begin{array}{lll}
I_{n \times n} & 0_{n \times(q-1) n}
\end{array}\right] M_{i}^{T}} & A_{0 r}^{T} Y_{i}^{T}+\delta(i, 0) C_{0 r}^{T} \bar{K}_{0}^{T}
\end{array}\right], \\
\Phi_{14}=\left[\begin{array}{c}
\bar{L}_{i}^{T} \\
0 \\
0
\end{array}\right], \\
\Phi_{22}:=-\left[\begin{array}{ccc}
Z_{i}+Z_{i}^{T} & 0 & Z_{i}+Y_{i}^{T}+S_{i}^{T} \\
* & M_{i}+M_{i}^{T} & 0 \\
* & * & Y_{i}+Y_{i}^{T}
\end{array}\right]+\mathscr{X}_{r}^{T} \mathcal{S}_{i},
\end{gathered}
$$




$$
\Phi_{23}=\left[\begin{array}{cc}
Z_{i} B_{w r} & Z_{i} B_{f r} \\
0 & 0 \\
Y_{i} B_{w r}+\bar{K}_{i} D_{r} & Y_{i} B_{f r}
\end{array}\right], \quad X_{r}=\left[\begin{array}{lll}
X_{(-1) r} & \cdots & X_{q r}
\end{array}\right]^{T},
$$

$\widehat{D}_{i r}$ is defined in (14) and $\mathcal{S}_{i}$ is the same in (21). Moreover, if (26) is feasible, the parameters of the desired robust fault detection filter can be given by

$$
G_{i}=V_{i}^{-1} \bar{G}_{i} S_{i}^{-1} V_{i}, \quad K_{i}=V_{i}^{-1} \bar{K}_{i}, \quad L_{i}=\bar{L}_{i} S_{i}^{-1} V_{i},
$$

where $V_{i} \in \mathbb{R}^{n \times n}$ is any invertible matrix (e.g., $V_{i}$ could be set as I).

Proof. Considering (20), Let $U_{i} \in \mathbb{R}^{n \times n}$ and $V_{i} \in \mathbb{R}^{n \times n}$ be two nonsingular matrices, and set

$$
Q_{i}^{T}=\left[\begin{array}{ccc}
Y_{i}^{T} & 0 & Q_{31 i}^{T} \\
0 & M_{i}^{T} & 0 \\
V_{i}^{T} & 0 & Q_{33 i}^{T}
\end{array}\right]
$$

Introducing the followind new matrices:

$$
\bar{Q}_{i}^{T}=\left[\begin{array}{ccc}
Z_{i}^{-T} & 0 & \bar{Q}_{31 i}^{T} \\
0 & I & 0 \\
U_{i}^{T} & 0 & \bar{Q}_{33 i}^{T}
\end{array}\right]
$$

where the entries $Q_{31 i}^{T}, Q_{33 i}^{T}, \bar{Q}_{31 i}^{T}$, and $\bar{Q}_{33 i}^{T}$ are uniquely determined from the following relation:

$$
\left[\begin{array}{cc}
Y_{i}^{T} & Q_{31 i}^{T} \\
V_{i}^{T} & Q_{33 i}^{T}
\end{array}\right]\left[\begin{array}{cc}
Z_{i}^{-T} & \bar{Q}_{31 i}^{T} \\
U_{i}^{T} & \bar{Q}_{33 i}^{T}
\end{array}\right]=\left[\begin{array}{cc}
Z_{i}^{-T} & \bar{Q}_{31 i}^{T} \\
U_{i}^{T} & \bar{Q}_{33 i}^{T}
\end{array}\right]\left[\begin{array}{cc}
Y_{i}^{T} & Q_{31 i}^{T} \\
V_{i}^{T} & Q_{33 i}^{T}
\end{array}\right]=I,
$$

we further have the following relation

$$
Q_{i}^{T} \bar{Q}_{i}^{T}=\bar{Q}_{i}^{T} Q_{i}^{T}=\left[\begin{array}{ccc}
I & 0 & 0 \\
0 & M_{i}^{T} & 0 \\
0 & 0 & I
\end{array}\right]
$$

By defining

$$
T_{i}=\left[\begin{array}{ccc}
Z_{i}^{T} & 0 & Y_{i}^{T} \\
0 & I & 0 \\
0 & 0 & V_{i}^{T}
\end{array}\right]
$$

we obtain

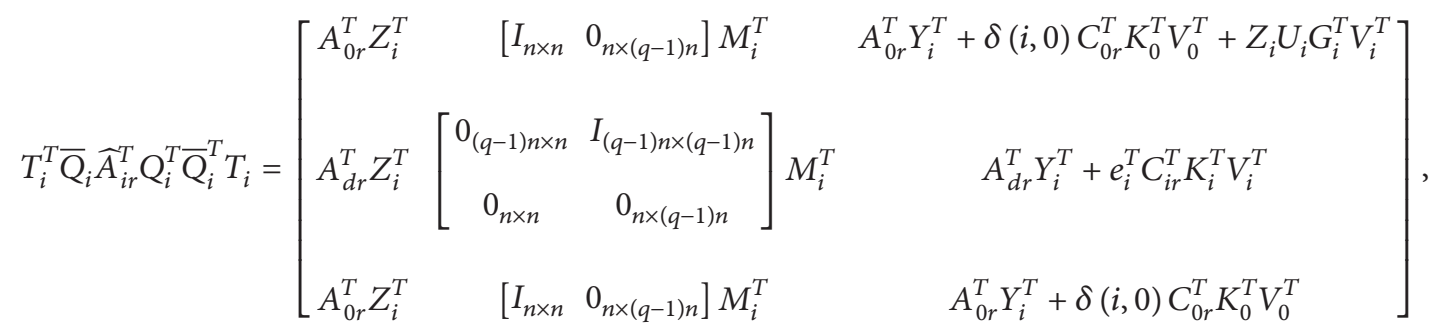

$$
\begin{aligned}
& T_{i}^{T} \bar{Q}_{i} \widehat{C}_{i}^{T}=\left[\begin{array}{c}
Z_{i} U_{i} L_{i}^{T} \\
0 \\
0
\end{array}\right], \quad T_{i}^{T} \bar{Q}_{i}\left(Q_{i}+Q_{i}^{T}\right) \bar{Q}_{i}^{T} T_{i}=\left[\begin{array}{ccc}
Z_{i}+Z_{i}^{T} & 0 & Z_{i}+Y_{i}^{T}+S_{i}^{T} \\
* & M_{i}+M_{i}^{T} & 0 \\
* & * & Y_{i}+Y_{i}^{T}
\end{array}\right], \\
& T_{i}^{T} \bar{Q}_{i} Q_{i} \widehat{B}_{i r}=\left[\begin{array}{cc}
Z_{i} B_{w r} & Z_{i} B_{f r} \\
0 & 0 \\
Y_{i} B_{w r}+V_{i} K_{i} D_{r} & Y_{i} B_{f r}
\end{array}\right] .
\end{aligned}
$$

Performing congruence transformations to (20) by $\operatorname{diag}\left\{\bar{Q}_{i}^{T} T_{i}, \bar{Q}_{i}^{T} T_{i}, I, I, I, I\right\}$, define

$$
X_{i r}=T_{i}^{T} \bar{Q}_{i} P_{i r} \bar{Q}_{i}^{T} T_{i}, \quad \bar{G}_{i}=V_{i} G_{i} U_{i}^{T} Z_{i}^{T}
$$

$$
\bar{K}_{i}=V_{i} K_{i}, \quad \bar{L}_{i}=L_{i} U_{i}^{T} Z_{i}^{T}, \quad S_{i}=V_{i} U_{i}^{T} Z_{i}^{T},
$$

then, it can be easily shown that LMIs (26) together with the additional constraints (29) and (30) are equivalent to LMIs 
in (20). Hence, if there exist matrices $X_{i r}>0, S_{i}, Z_{i}, Y_{i}, \bar{G}_{i}$, $\bar{K}_{i}, \bar{L}_{i}, M_{i}, i=-1, \ldots, q$, and $P_{t}>0$ such that LMIs (20) are feasible, the overall fault detection dynamic (11) is robust mean square stable and the constraint (16) is satisfied.

Furthermore, from LMIs (26), we have for $i=-1, \ldots, q$,

$$
\left[\begin{array}{ccc}
Z_{i}+Z_{i}^{T} & 0 & Z_{i}+Y_{i}^{T}+S_{i}^{T} \\
* & M_{i}+M_{i}^{T} & 0 \\
* & * & Y_{i}+Y_{i}^{T}
\end{array}\right]>0
$$

This indicates that $Z_{i}$ and $Y_{i}$ are nonsingular and

$$
\begin{aligned}
& {\left[\begin{array}{lll}
I & 0 & -I
\end{array}\right]\left[\begin{array}{ccc}
Z_{i}+Z_{i}^{T} & 0 & Z_{i}+Y_{i}^{T}+S_{i}^{T} \\
* & M_{i}+M_{i}^{T} & 0 \\
* & * & Y_{i}+Y_{i}^{T}
\end{array}\right]\left[\begin{array}{c}
I \\
0 \\
-I
\end{array}\right]} \\
& \quad=-S_{i}-S_{i}^{T}>0,
\end{aligned}
$$

which implies that $S_{i}$ is nonsingular and also ensures the existence of parameter matrices $G_{i}, K_{i}$, and $L_{i}$ in (28). The proof is completed.

Remark 10. In Theorem 9, uncertainty-dependent robust fault detection filter design result is provided, which reduces the conservatism than the uncertainty-independent results. If we impose

$$
X_{\text {ir }}=X_{i}, \quad i=-1, \ldots, q, \quad r=1, \ldots, v,
$$

to (26), the uncertainty-independent result can be recovered.

Remark 11. In most cases, we can know the size of the measurement delay or whether the data is missing at a certain time by using the time-stamp at the system node [5], and therefore the jumping parameters of the transformed MJS are accessible. In this sense, Theorem 9 provides us with network-status-dependent fault detection filter design methods. On the other hand, if the network status is not accessible; that is, the jumping parameters of the transformed MJS are unavailable, a network-status-independent result can be easily obtained by imposing

$$
\begin{gathered}
S_{i}=S, \quad \bar{G}_{i}=\bar{G}, \\
\bar{K}_{i}=\bar{K}, \quad \bar{L}_{i}=\bar{L}, \quad i=-1, \ldots, q,
\end{gathered}
$$

in Theorem 9.

Note that (26) are LMIs over both the matrix variables and the prescribed scalar $\gamma^{2}$. This implies that (i) the robust fullorder fault detection filter can be obtained from the solution of convex optimization problems in terms of LMIs, which can be solved via efficient interior-point algorithms [23]; (ii) the scalar $\gamma^{2}$ can be included as one of the optimization variables for LMIs (20), which makes it possible to obtain the minimum noise attenuation level bound for the fault detection dynamics (11). Then, the uncertainty-dependent suboptimal robust fault detection filter can be readily found by solving the following convex optimization problem.
Problem 12. Consider the parameter uncertainty (5), the suboptimal robust fault detection filter for networked systems (1) with multiple state-delays and unknown inputs based on the idea of uncertainty dependence and network status dependence can be brought forward as follows:

$$
\min _{\substack{X_{i r}>0, S_{i}, Z_{i}, Y_{i}, M_{i}, \bar{G}_{i}, \bar{K}_{i}, \bar{L}_{i}, P_{t}>0, i=-1, \ldots, q, r=1, \ldots, v}} \gamma^{2}, \quad \text { s.t. (26). }
$$

For the problems mentioned above, the parameters of the sub-optimal robust fault detection filter can be determined by (28), and the sub-optimal robust $H_{\infty}$ attenuation level for fault detection dynamics is given by $\gamma^{*}=\sqrt{\gamma_{\text {opt }}^{2}}$, where $\gamma_{\text {opt }}^{2}$ are the sub-optimal solution of the corresponding convex optimization problems.

Here is a summary of the whole fault detection method for system (1).

Step 1. Determine the vertex of uncertain parameters of (1).

Step 2. Calculate the parameters of fault detection filter using Theorem 9.

Step 3. Get a appropriate threshold from experiments for a specific noise type.

Step 4. Generate a real-time residual signal from the fault detection filter designed in Step 2.

Step 5. Compare the evaluation function with the threshold and use the logic (19) to alarm a fault.

Remark 13. In the present work, a model-based approach is considered since there is a mathematical model for the system plant. However, when there is no such a model and only input and output data can be obtained in many complex systems, data-driven methods may work better than the model based ones since there is a leakage of prior information of system dynamics. Please see $[24,25]$ for typical data-driven fault detection methods.

\section{A Numerical Example}

To illustrate the effectiveness of the proposed method, we provide a numerical examples in this section. Consider system (1) with the following uncertain system parameters:

$$
\begin{gathered}
A_{0}=\left[\begin{array}{cc}
0 & 0.5 \\
0.2 & \theta
\end{array}\right], \quad A_{1}=\left[\begin{array}{cc}
0.2 & 0 \\
0.7 & 0.1
\end{array}\right], \\
B_{w}=\left[\begin{array}{l}
0.5 \\
0.3
\end{array}\right], \quad B_{f}=\left[\begin{array}{c}
-1 \\
2
\end{array}\right], \\
C_{0}=C_{1}=\left[\begin{array}{cc}
0.2 & 0 \\
0 & 0.5
\end{array}\right], \quad D=\left[\begin{array}{c}
0.2 \\
-0.1
\end{array}\right],
\end{gathered}
$$

where $\theta$ is an uncertain real parameter satisfying $0.1 \leq \theta \leq$ 0.3 . The initial state values $\varphi_{k}$ are set to be $\varphi_{-1}=\varphi_{0}=0$. 
Let $q=1$, so the state-space of the Markov chain $\left\{\tau_{k}\right\}$ is $\Xi=$ $\{-1,0,1\}$. The transition probability matrix is given by

$$
\Lambda:=\left[\begin{array}{ccc}
\lambda_{-1,-1} & \lambda_{-1,0} & \lambda_{-1,1} \\
\lambda_{0,-1} & \lambda_{0,0} & \lambda_{0,1} \\
\lambda_{1,-1} & \lambda_{1,0} & \lambda_{1,1}
\end{array}\right]=\left[\begin{array}{lll}
0.7 & 0.2 & 0.1 \\
0.3 & 0.4 & 0.3 \\
0.2 & 0.3 & 0.5
\end{array}\right],
$$

and the initial mode is set to be $\tau_{0}=0$. For $k=0,1, \ldots, 300$, the unknown input $w_{k}$ is supposed to be a random noise uniformly distributed over $[-0.5,0.5]$, and the fault signal $f_{k}$ is of the following intermittent form:

$$
f_{k}= \begin{cases}1, & \text { for } k=200 s+101, \ldots, 200 s+200(s=0,1,2), \\ 0, & \text { others. }\end{cases}
$$

The weighting matrix is supposed to be $T_{f}(z)=(0.5 z) /$ $(z-0.5)$, with the following state space realization:

$$
\begin{gathered}
\widehat{x}_{k+1}=0.5 \widehat{x}_{k}+0.25 f_{k}, \\
\widehat{f}_{k}=\widehat{x}_{k}+0.5 f_{k}, \\
\widehat{x}_{0}=0,
\end{gathered}
$$

where $f_{k}$ and $\widehat{f}_{k}$ are shown in Figure 1.

With the predefined parameters, from Theorem 9, Problem 12 can be solved by using the Matlab LMI toolbox [23]. As a result, the minimum noise attenuation level bound of the fault detection dynamic is $\gamma_{\mathrm{opt}}=1.0012$, and the parameters of the sub-optimal fault detection filter in different modes are given by

$$
\begin{aligned}
& G_{-1}=\left[\begin{array}{ll}
0.1311 & 1.3402 \\
0.1620 & 0.6100
\end{array}\right], \quad K_{-1}=\left[\begin{array}{cc}
4.3089 & 8.6222 \\
21.7929 & 43.5879
\end{array}\right], \\
& L_{-1}=\left[\begin{array}{ll}
-0.7029 & 0.0724
\end{array}\right], \quad G_{0}=\left[\begin{array}{cc}
0.0315 & 0.4950 \\
-0.0373 & 0.2187
\end{array}\right] \text {, } \\
& K_{0}=\left[\begin{array}{rr}
-0.0002 & -0.0025 \\
-0.0005 & -0.0006
\end{array}\right], \quad L_{0}=\left[\begin{array}{ll}
-0.1088 & -0.0396
\end{array}\right] \text {, } \\
& G_{1}=\left[\begin{array}{ll}
-0.1343 & 0.8207 \\
-0.0807 & 0.3832
\end{array}\right], \quad K_{1}=\left[\begin{array}{rr}
-0.0014 & -0.0001 \\
-0.0018 & -0.0002
\end{array}\right] \text {, } \\
& L_{1}=\left[\begin{array}{ll}
-0.6086 & 0.2436
\end{array}\right] \text {. }
\end{aligned}
$$

If we impose the uncertainty-independent and networkstatus-independent method indicated in Remarks 10 and 11, a fault detection filter with $\gamma_{\mathrm{opt}}=1.0082$ can be obtained.

Next, we consider the time-domain simulation using the obtained fault detection filter. we arbitrarily choose $\theta=0.15$. Figure 2 shows the measurement mode with random delays and stochastic missing phenomenon. $\tau_{k}=-1,0,1$, means that the measurement is missing, transmitted over the network ideally, and with one-step delay, respectively.

Figure 3 shows the generated residual signal $r_{k}$, and the evolution of $J^{L}(k)$ is presented in Figure 4, where we choose

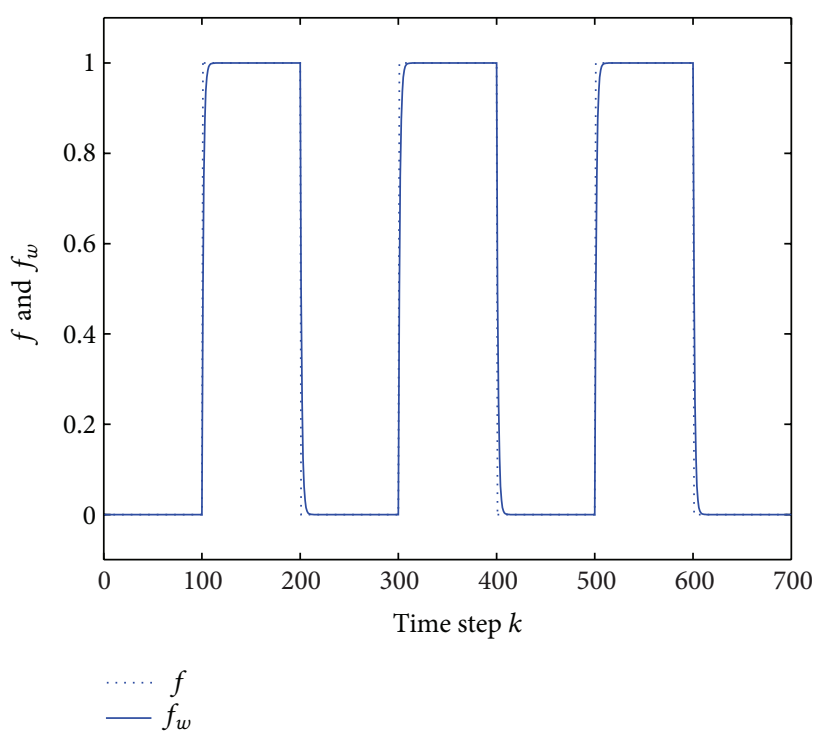

FIGURE 1: Fault $f_{k}$ and weighting fault $\widehat{f}_{k}\left(f_{w}\right)$.

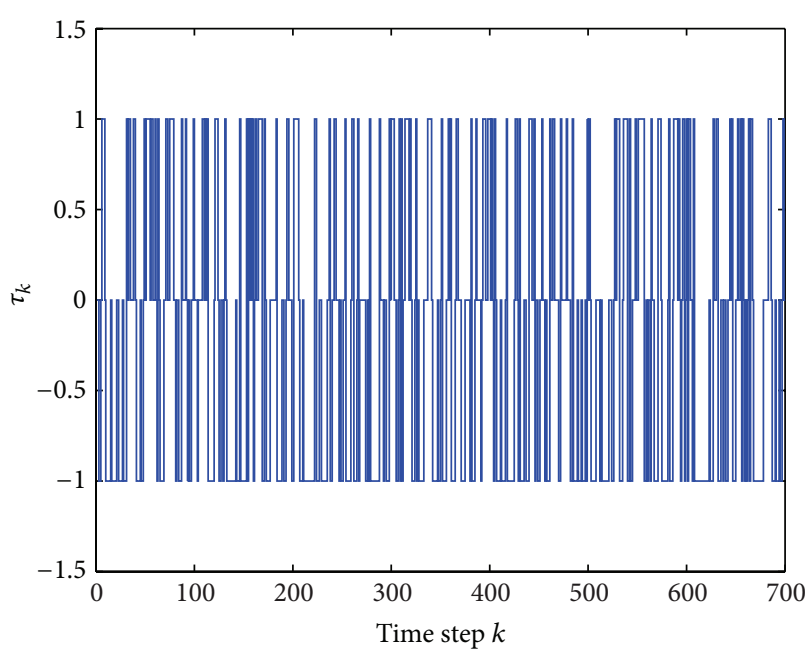

FIgURE 2: Measurement mode over network.

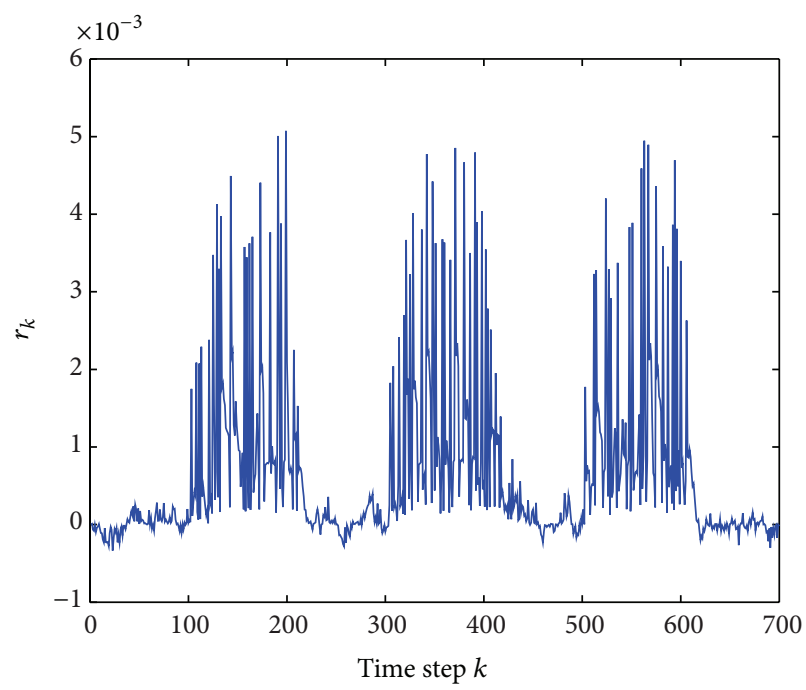

FIGURE 3: Residual signal $r_{k}$. 


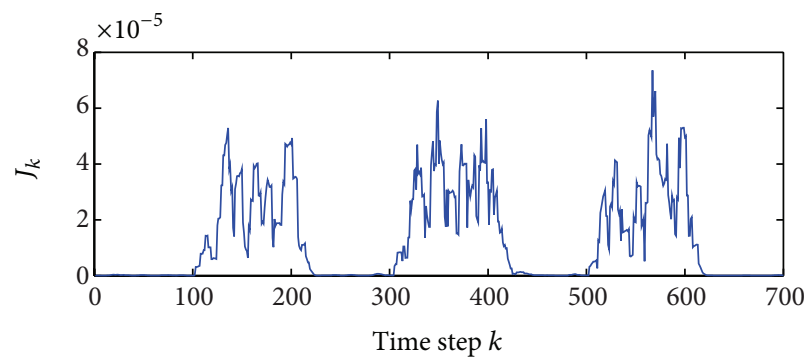

(a)

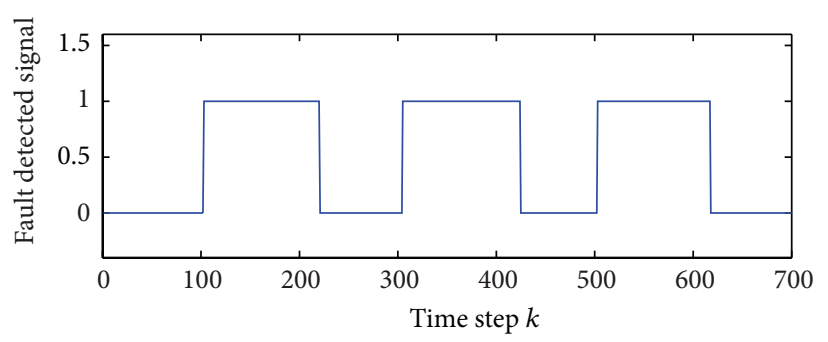

(b)

FIGURE 4: Evolution of $J^{L}(k)$.

the length of time window $L=8$. After 400 times of simulations, we get a threshold $J_{\text {th }}^{L}=1.5215 \times 10^{-6}$. Figure 4 shows the real-time fault detection result:

$$
\begin{aligned}
& 4.9199 \times 10^{-8}=J(102)<J_{\text {th }}^{L}<J(103)=3.1041 \times 10^{-6}, \\
& 1.9392 \times 10^{-6}=J(220)<J_{\text {th }}^{L}<J(221)=1.4006 \times 10^{-6}, \\
& 9.1655 \times 10^{-8}=J(304)<J_{\text {th }}^{L}<J(305)=3.4154 \times 10^{-6}, \\
& 2.5346 \times 10^{-6}=J(424)<J_{\text {th }}^{L}<J(425)=6.0104 \times 10^{-7}, \\
& 8.1835 \times 10^{-8}=J(502)<J_{\text {th }}^{L}<J(503)=3.2139 \times 10^{-6}, \\
& 1.9241 \times 10^{-6}=J(617)<J_{\text {th }}^{L}<J(618)=1.1692 \times 10^{-6} .
\end{aligned}
$$

From above inequations, we can observe the fault occurrence can be detected after 3, 5, 3 steps, while the disappearance of fault can be detected after 21, 25, 18 steps, respectively.

Remark 14. After introducing a novel residual evaluation function of the incremental type (17), one can detect not only the occurrence of a fault but also its disappearance. It can be observed from Figure 4 that the disappearance of the fault usually needs longer time to detect than the occurrence of faults. This is because the mathematical model of the system is based on the plant without the faults. This is of engineering significance and the proposed method in this paper can be applied to detect intermittent faults in many practical industrial processes.

Remark 15. The length of time window for evaluation function $L$ in (17) is also a factor that can affect the fault detection performance. Longer $L$ can reduce the missing alarm rate and false alarm rate; however, it costs longer detection time. Short $L$ can provide a rapid fault detection but this results in larger missing alarm rate and false alarm rate.

\section{Conclusions}

In this paper, the robust intermittent fault detection problem has been investigated for a class of discrete-time uncertain networked systems with state delays and incomplete measurements. The random delay and stochastic missing phenomenon in the measurements have been simultaneously investigated. Polytopic-type parameter uncertainty in the state-space model matrices has been considered. By augmenting the states, the addressed robust fault detection problem has been converted to an auxiliary robust $H_{\infty}$ filtering problem for a certain Markovian jumping system (MJS). A sufficient condition for the design of the desired robust fault detection filter has been established. A numerical example has been introduced to illustrate the effectiveness of the proposed methodology.

\section{Acknowledgments}

This work was supported in part by the National Natural Science Foundation of China under Grant 61074084 and 61074085 and an open Grant of the Key Laboratory of HydroScience and Engineering in Chongqing Jiaotong University under Grant SLK2010.

\section{References}

[1] S. Elmadssia, K. Saadaoui, and M. Benrejeb, "New delaydependent stability conditions for linear systems with delay," Systems Science and Control Engineering, vol. 1, no. 1, pp. 2-11, 2013.

[2] Y. Chen and K. A. Hoo, "Stability analysis for closed-loop management of a reservoir based on identification of reduced-order nonlinear model," Systems Science and Control Engineering, vol. 1, no. 1, pp. 12-19, 2013.

[3] M. Darouach and M. Chadli, "Admissibility and control of switched discrete-time singular systems," Systems Science and Control Engineering, vol. 1, no. 1, pp. 43-51, 2013.

[4] W. Zhang, M. S. Branicky, and S. M. Phillips, "Stability of networked control systems," IEEE Control Systems Magazine, vol. 21, no. 1, pp. 84-99, 2001.

[5] J. Nilsson, Real-time control systems with delays [Ph.D. dissertation], Department Automatic Control, Lund Institute of Technology, Lund, Sweden, 1998.

[6] D. Yue, E. Tian, and Q.-L. Han, "A delay system method for designing event-triggered controllers of networked control systems," IEEE Transactions on Automatic Control, vol. 58, no. 2, pp. 475-481, 2013.

[7] P. M. Frank, S. X. Ding, and B. Koppen-Seliger, "Current developments in the theory of FDI," in Proceedings of the 4th IFAC Symposium Fault Detection Supervision Safety Technical Processes, Proceedings of SAFEPRO-CESS, pp. 16-27, Budapest, Hungary, 2000. 
[8] M. Kinnaert, "Fault diagnosis based on analytical models for linear and nonlinear systems," in Proceedings of the 15th International Workshop on Principles of Diagnosis, Proceedings of SAFEPROCESS, pp. 37-50, Washington, DC, USA, 2003.

[9] S. X. Ding, T. Jeinsch, P. M. Frank, and E. L. Ding, "A unified approach to the optimization of fault detection systems," International Journal of Adaptive Control and Signal Processing, vol. 14, no. 7, pp. 725-745, 2000.

[10] M. Zhong, H. Ye, P. Shi, and G. Wang, "Fault detection for markovian jump systems," IEE Proceedings Control Theory and Applications, vol. 152, no. 4, pp. 397-402, 2005.

[11] H. Fang, H. Ye, and M. Zhong, "Fault diagnosis of networked control systems (MED '09)," in Mediterranean Conference on Control and Automation, Proceedings of SAFEPROCESS, pp. 112, Beijing, China, June 2006.

[12] M. Nader and K. Khashayar, "Actuator fault detection and isolation for a network of unmanned vehicles," IEEE Transactions on Automatic Control, vol. 54, no. 4, pp. 835-840, 2009.

[13] R. Luck and A. Ray, "An observer-based compensator for distributed delays," Automatica, vol. 26, no. 5, pp. 903-908, 1990.

[14] L. Zhang, Y. Shi, T. Chen, and B. Huang, "A new method for stabilization of networked control systems with random delays," IEEE Transactions on Automatic Control, vol. 50, no. 8, pp. 11771181, 2005.

[15] F. Yang, Z. Wang, Y. S. Hung, and M. Gani, “ $H_{\infty}$ control for networked systems with random communication delays," IEEE Transactions on Automatic Control, vol. 51, no. 3, pp. 511-518, 2006.

[16] Z. Wang, D. W. C. Ho, and X. Liu, "Variance-constrained filtering for uncertain stochastic systems with missing measurements," IEEE Transactions on Automatic Control, vol. 48, no. 7, pp. 1254-1258, 2003.

[17] X. He, Z. Wang, and D. Zhou, "Robust $H_{\infty}$ filtering for networked systems with multiple state-delays," International Journal of Control, vol. 80, no. 8, pp. 1217-1232, 2007.

[18] P. Zhang, S. X. Ding, P. M. Frank, and M. Sader, "Fault detection of networked control systems with missing measurements," in Proceedings of Asian Control Conference, pp. 1258-1263, Melbourne, Australia, 2004.

[19] O. Contant, S. Lafortune, and D. Teneketzis, "Diagnosis of intermittent faults," Discrete Event Dynamic Systems: Theory and Applications, vol. 14, no. 2, pp. 171-202, 2004.

[20] O. L. V. Costa and M. D. Fragoso, "Stability results for discretetime linear systems with Markovian jumping parameters," Journal of Mathematical Analysis and Applications, vol. 179, no. 1, pp. 154-178, 1993.

[21] O. L. V. Costa and R. P. Marques, "Mixed $H_{2} / H_{\infty}$-control of discrete-time Markovian jump linear systems," IEEE Transactions on Automatic Control, vol. 43, no. 1, pp. 95-100, 1998.

[22] M. C. de Oliveira, J. C. Geromel, and J. Bernussou, "Extended $\mathrm{H}_{2}$ and $H_{\infty}$ norm characterizations and controller parametrizations for discrete-time systems," International Journal of Control, vol. 75, no. 9, pp. 666-679, 2002.

[23] S. Boyd, L. El Ghaoui, E. Feron, and V. Balakrishnan, Linear Matrix Inequalities in System and Control Theory, vol. 15 of SIAM Studies in Applied Mathematics, Society for Industrial and Applied Mathematics (SIAM), Philadelphia, PA, USA, 1994.

[24] S. Yin, H. Luo, and S. X. Ding, "Real-time implementation of fault tolerant control system with performance optimization," IEEE Transactions on Industrial Electronics, 2013.
[25] S. Yin, S. X. Ding, A. Gaghani, H. Hao, and P. Zhang, "A comparison study of basic data-driven fault diagnosis and process monitoring methods on the benchmark Tennessee Eastman process," Journal of Process Control, vol. 22, no. 9, pp. 1567-1581, 2012. 


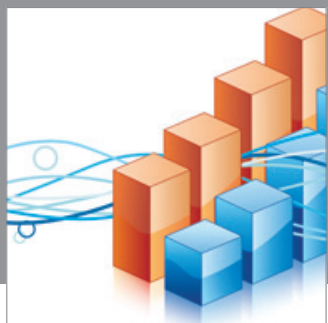

Advances in

Operations Research

mansans

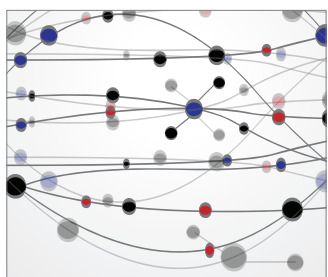

The Scientific World Journal
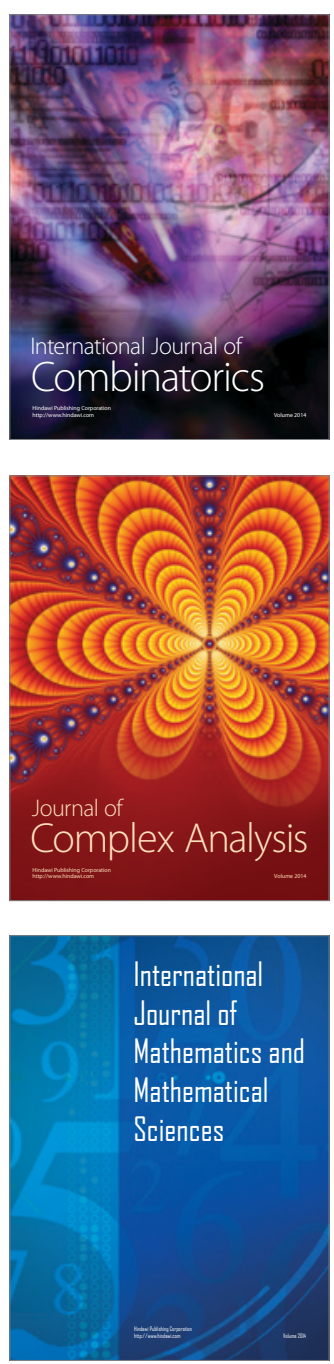
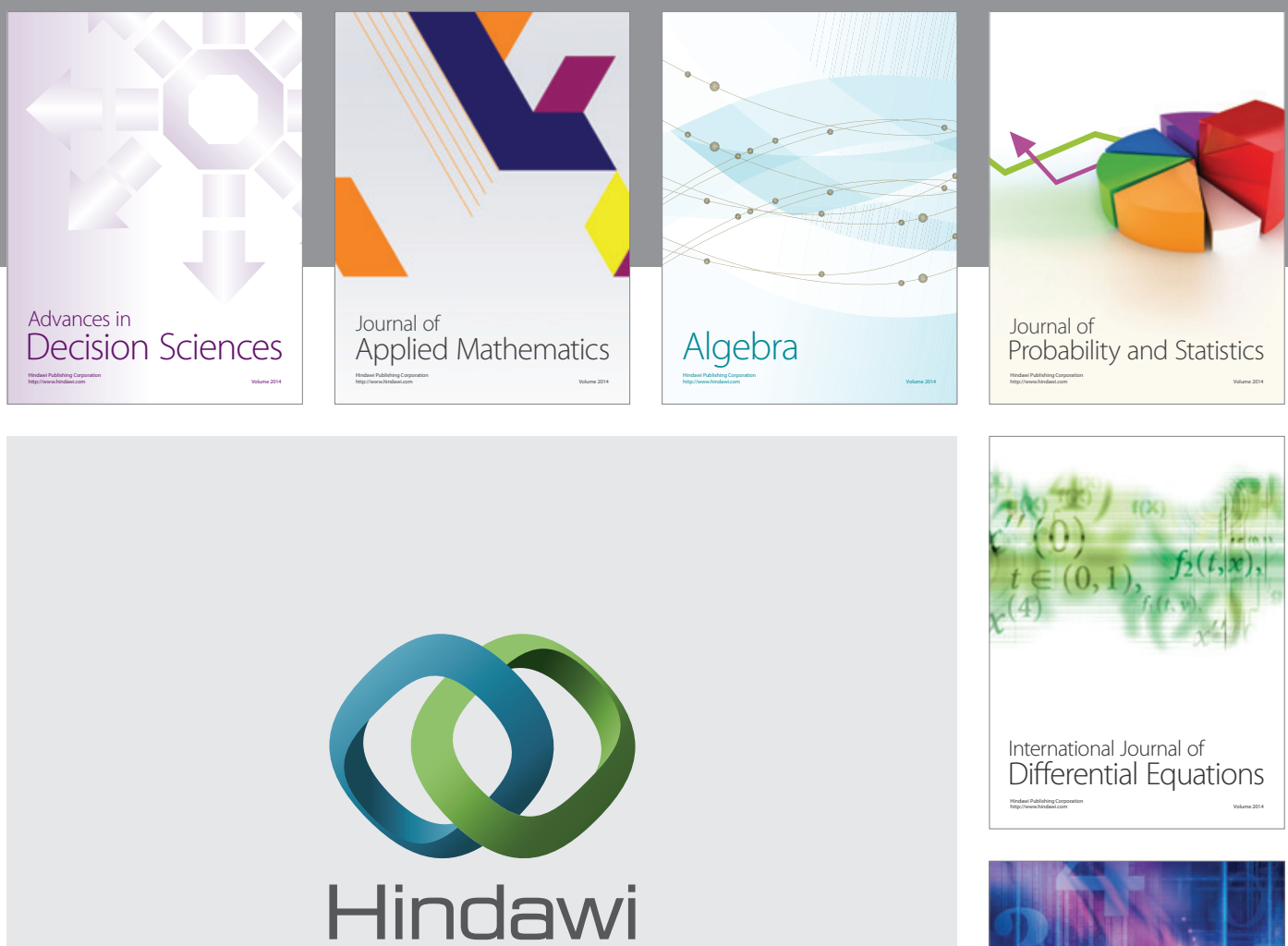

Submit your manuscripts at http://www.hindawi.com
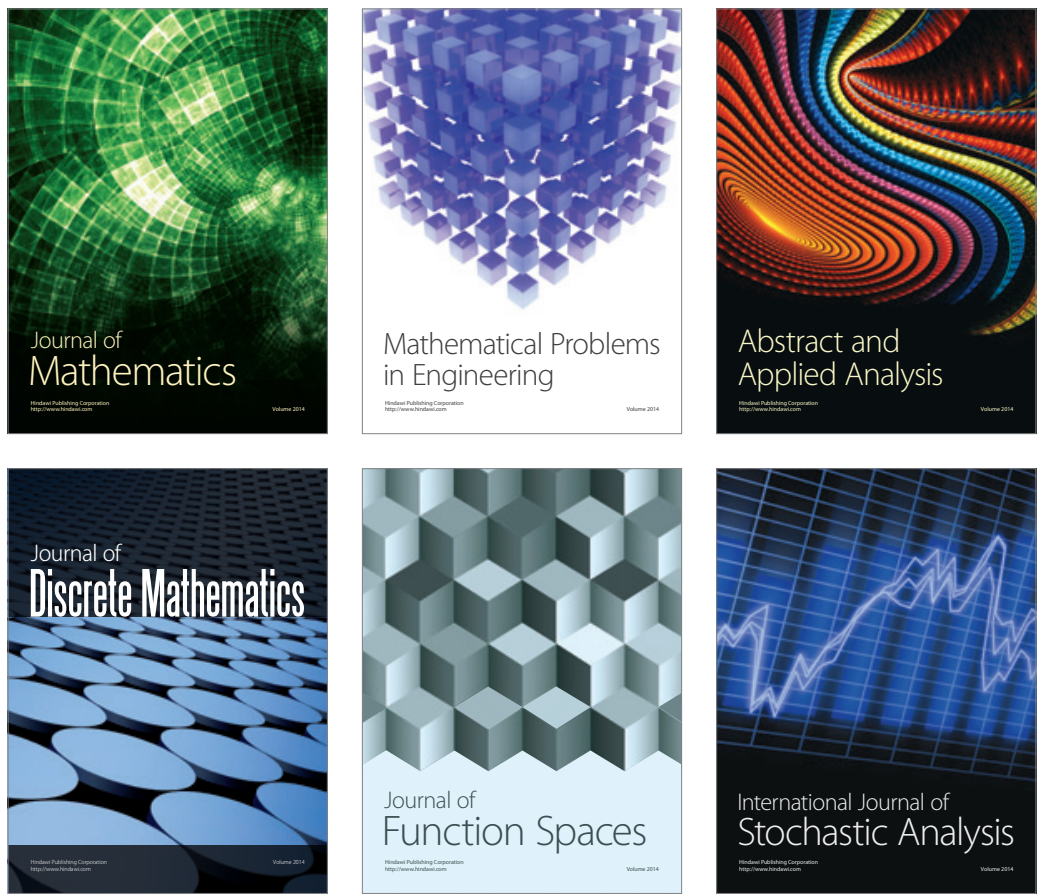

Journal of

Function Spaces

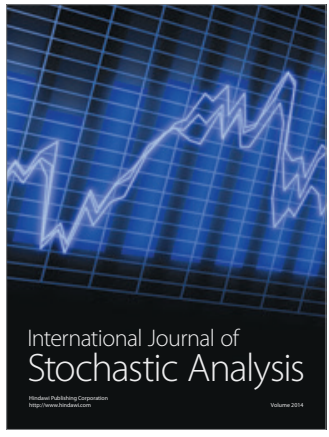

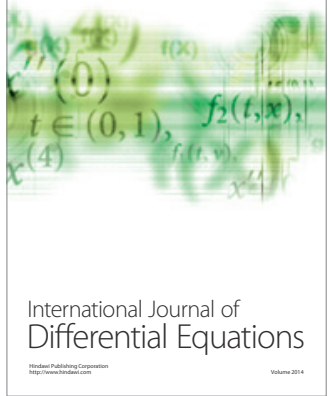
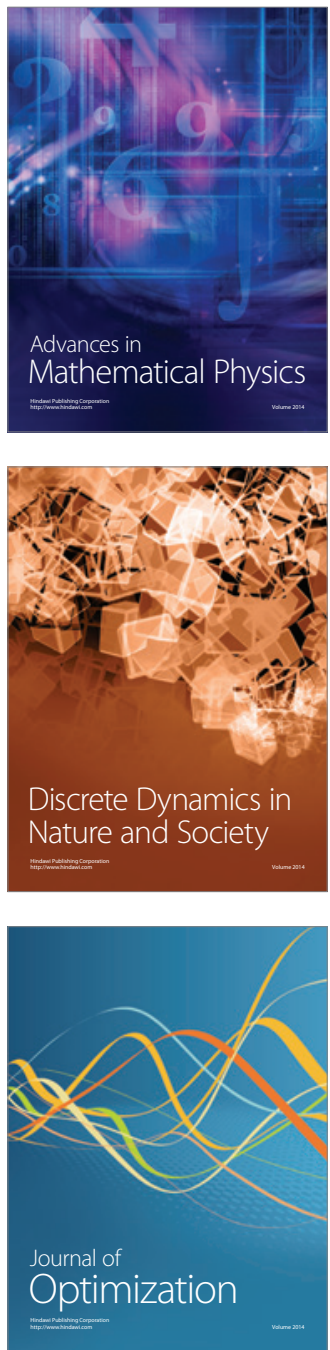